\title{
miR-29s function as tumor suppressors in gliomas by targeting TRAF4 and predict patient prognosis
}

\author{
Cuijuan Shi ${ }^{1,2,3}$, Chun Rao ${ }^{1,2,3}$, Cuiyun Sun ${ }^{1,2,3}$, Lin Yu4 ${ }^{4}$ Xuexia Zhou ${ }^{1,2,3}$, Dan Hua ${ }^{1,2,3}$, Run Wang ${ }^{1,2,3}$, Wenjun Luo ${ }^{1,2,3}$,
} Zhendong Jiang ${ }^{1,2,3}$, Junhu Zhou ${ }^{2,3,5}$, Qian Wang ${ }^{1,2,3}$ and Shizhu Yu ${ }^{1,2,3}$

\begin{abstract}
Robust proliferation and apoptosis inhibition of tumor cells are responsible for the high mortality and poor outcome of patients with high-grade gliomas. miR-29a/b/c have been reported to be important suppressors in several human tumor types. However, their exact roles in gliomagenesis and their relevance to patient prognosis remain unclear. In this study, using 187 human glioma specimens and 20 nontumoral brain tissues, we demonstrated that the expression of miR-29a/b/c decreased progressively as the grade of glioma and the Ki-67 index increased. However, the expression of TRAF4, the functional target of miR-29a/b/c, exhibited the inverse trend, and its level was inversely correlated with the levels of miR-29a/b/c. A Kaplan-Meier analysis demonstrated that the miR-29a/b/c and TRAF4 levels were closely associated with patient survival even in patients with the same tumor grade and identical IDH gene status. A functional study verified that miR-29a/b/c induced apoptosis and suppressed the proliferation of glioma cells by directly targeting TRAF4. An investigation of the mechanism revealed that miR-29a/b/c promoted apoptosis through the TRAF4/AKT/MDM2 pathway in a p53-dependent manner, while miR-29a/b/c induced G1 arrest and inhibited tumor cell proliferation by blocking the phosphorylation of AKT and GSK-3ß, and the expression of cyclin D1 and cMyc. Furthermore, TRAF4-knockdown perfectly simulated the anti-glioma effects of miR-29a/b/c. These findings enrich our understanding of gliomagenesis, highlight the prognostic value of miR-29a/b/c and TRAF4, and imply their potential therapeutic roles in malignant gliomas.
\end{abstract}

\section{Introduction}

Gliomas are the most frequent primary brain tumors in adults $^{1,2}$, and malignant gliomas, especially glioblastomas, are aggressive and lethal neoplasms characterized by rapid growth and persistent infiltration, which means that a radical resection is almost impossible ${ }^{3}$. Although unlimited proliferation and inhibition of tumor cell apoptosis have been accepted as the key reasons for the rapid

\footnotetext{
Correspondence: Qian Wang (wangqiantni@163.com) or

Shizhu Yu (tjyushizhu@163.com)

${ }^{1}$ Department of Neuropathology, Tianjin Neurological Institute, Tianjin Medical University General Hospital, Tianjin 300052, China

${ }^{2}$ Tianjin Key Laboratory of Injuries, Variations and Regeneration of the Nervous System, Tianjin 300052, China

Full list of author information is available at the end of the article.

Edited by A. Peschiaroli
}

growth of malignant gliomas, the underlying epigenetic and genetic changes are still poorly understood ${ }^{4}$. Moreover, prognostic biomarkers and therapeutic targets for gliomas have not been fully characterized. In addition, a thorough study on the molecular mechanisms of glioma formation and malignant progression is a prerequisite for the screening of valuable diagnostic and prognostic biomarkers and for the optimization of the therapeutic strategies against malignant gliomas.

Recent studies have demonstrated that miRNAs are important epigenetic regulators in tumorigenesis and promising biomarkers for prognosis ${ }^{5-10}$. The human miR29 family consists of three closely related members, miR$29 \mathrm{a}, \mathrm{b}$, and $\mathrm{c}^{11}$. The abnormal decrease in their levels and the association of that decrease with a poor prognosis

\section{(c) The Author(s) 2018}

(c) (i) Open Access This article is licensed under a Creative Commons Attribution 4.0 International License, which permits use, sharing, adaptation, distribution and reproduction cc) in any medium or format, as long as you give appropriate credit to the original author(s) and the source, provide a link to the Creative Commons license, and indicate if changes were made. The images or other third party material in this article are included in the article's Creative Commons license, unless indicated otherwise in a credit line to the material. If material is not included in the article's Creative Commons license and your intended use is not permitted by statutory regulation or exceeds the permitted use, you will need to obtain permission directly from the copyright holder. To view a copy of this license, visit http://creativecommons.org/licenses/by/4.0/. 
have been reported in various malignancies ${ }^{12-20}$. Moreover, recent efforts on the manipulation of exogenous miR-29 family members represent appealing approaches to anti-tumor therapy ${ }^{17,21}$, which suggests that miR-29a/ $\mathrm{b} / \mathrm{c}$ can be used as prognostic biomarkers and that they are therapeutic candidates for these tumors. However, their prognostic relevance and tumor suppressive effects still need to be fully elucidated in gliomas.

Tumor necrosis factor receptor-associated factor-4 (TRAF4), which is a cytoplasmic adaptor that functions as an E3 ubiquitin ligase, has been shown to be overexpressed in several malignancies and to participate in tumorigenic processes ${ }^{22-24}$. Two previous studies have reported that TRAF4 was a natural target of miR-29 in metastatic prostate cancer and human fetal lung fibroblast IMR-90 cells $^{24,25}$. However, to the best of our knowledge, the expression pattern of TRAF4 in gliomas and its exact roles in gliomagenesis remain largely elusive.

In the present study, we demonstrated that $\mathrm{miR}-29 \mathrm{a} / \mathrm{b} / \mathrm{c}$ induced glioma cell apoptosis through the TRAF4/AKT/ MDM2 pathway in a p53-dependent manner, and restrained cell proliferation by directly targeting the TRAF4/AKT/GSK-3 $\beta$ pathway. Our results also implied the potential value of miR-29a/b/c and TRAF4 in the prognosis of glioma patients and as potential therapies for malignant gliomas.

\section{Materials and methods}

\section{Tissue samples and clinical data}

In all, 187 surgical specimens of human astrocytic gliomas and 20 nontumoral brain tissues were collected form Tianjin Medical University General Hospital (TMUGH) and were included in the present study after the patients provided written consent. The specimens were fixed in $3.7 \%$ buffered formaldehyde immediately after surgical excision and were embedded in paraffin (FFPE samples). Then, 5 - $\mu \mathrm{m}$-thick serial tissue sections were prepared for hematoxylin and eosin staining, miR$29 \mathrm{a} / \mathrm{b} / \mathrm{c}$ in situ hybridization, and immunohistochemistry (IHC) for TRAF4 and Ki-67. The pathologic diagnoses were rendered independently by two neuropathologists according to the 2016 World Health Organization (WHO) classification of central nervous system tumors ${ }^{1}$. The clinicopathologic features, including the WHO grades, $I D H 1 / 2$ gene statuses, and KPS scores, are summarized in Supplementary Table 1. All 187 glioma patients with complete clinical information were followed-up from the date of operation until 31 December 2014; the follow-up time ranged from 3.6 to 88.3 months.

Independent RNA-seq data of 638 human glioma samples were obtained from the Cancer Genome Atlas (TCGA) database (https://cancergenome.nih.gov/). The expression levels of TRAF4 were measured using the Illumina HiSeq RNA Sequencing platform. After $\log _{2}$ transformation, the expression data were subjected to a Kaplan-Meier analysis to verify the relationships between the TRAF4 levels and the overall survival (OS) (638 cases) and disease-free survival (DFS) (501 cases) of the glioma patients. For Oncomine data analyses (http://www. oncomine.org), the database was searched for TRAF4 using the following filter setting: Cancer vs. Normal analysis in brain and CNS cancer, $P$-value of $<1 \times 10^{-4}$, and gene rank in the top $10 \%$. The values from each published data set were linked to the graphical representations of the original data. TRAF4 mRNA levels were compared between human glioblastoma tissues and normal brain tissues (control) by Student's $t$-test.

\section{In situ hybridization and IHC}

In situ hybridization (ISH) and IHC were performed as previously described ${ }^{6,7}$. For ISH, the locked nucleic acidmodified and digoxin-labeled probes for miR-29a/b/c and the control oligonucleotide with scrambled sequence (Scr) were purchased from TaKaRa (Dalian, China; Supplementary Table 2). The rhodamine (TRITC)-conjugated anti-digoxin antibody and 4',6-diamidino-2-phenylindole were purchased form Roche (Indianapolis, IN, USA). IHC staining was performed with primary antibodies including a rabbit anti-human TRAF4 antibody (Santa Cruz Biotechnology, Santa Cruz, CA, USA) and a rabbit antihuman Ki-67 antibody (Millipore, Billerica, MA, USA). ISH and IHC images were acquired with a DM6000B fluorescence microscope (Leica, Wetzlar, Germany). For each slice, the number of cells in six randomly selected $\times 400$ microscopic fields was counted with Image-Pro Plus 5.0 (Leica, Wetzlar, Germany), and the labeling index (LI $\%$ ) was calculated as the proportion of positive cells.

\section{Cell cultures, lentiviruses, and stable sub-cell lines}

The human glioblastoma cell lines U87MG and DBTRG-05MG were obtained from the American Type Culture Collection (ATCC, Manassas, VA, USA), while the SNB19, U251, and LN308 cell lines were purchased from the China Academia Sinica Cell Repository (Shanghai, China). The TJ905 cell line, which was derived from a Chinese patient with glioblastoma, was established and maintained by our lab. The immortalized human astrocyte cell line UC2 was used as the nontumoral control. The DBTRG-05MG cell line was maintained in RPMI-1640 (Gibco, Grand Island, NY, USA), while the others were maintained in Dulbecco's modified Eagle's medium (Gibco, Grand Island, NY, USA). All the media were supplemented with $10 \%$ fetal bovine serum (Gibco, Grand Island, NY, USA), and the cells were cultured at $37^{\circ} \mathrm{C}$ in a humidified atmosphere containing $5 \% \mathrm{CO}_{2}$. The recombinant lentiviruses expressing miR-29a, miR-29b, miR-29c, or the scrambled control (Scr) were constructed and packaged by 


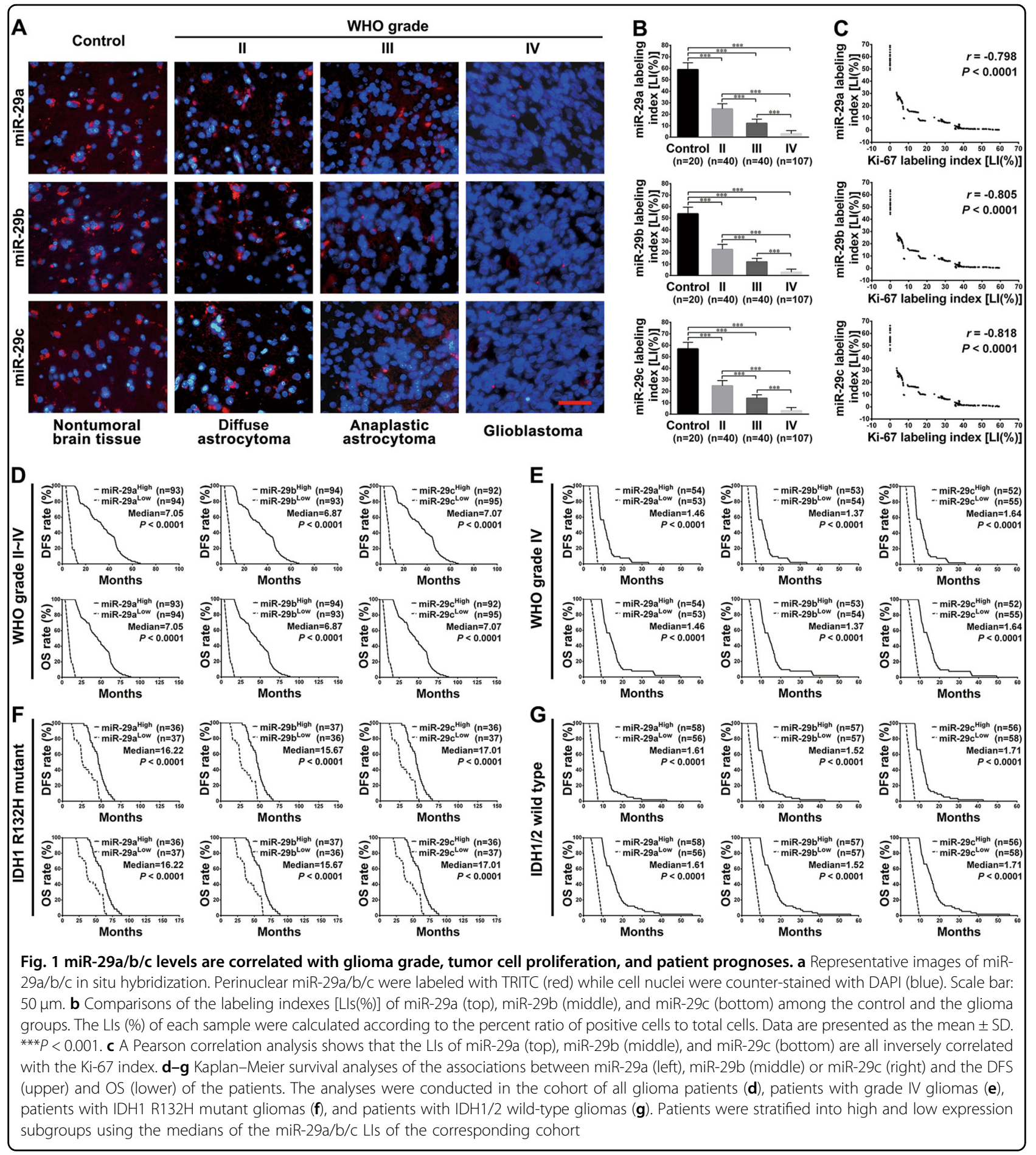

Genechem (Shanghai, China). The corresponding subcell lines of U87MG, DBTRG-05MG, SNB19, and U251 were established by lentivirus infection and puromycin selection. The expression efficiencies of the exogenous miRNAs were quantified by quantitative reverse transcriptase-PCR (qRT-PCR).
Oligonucleotides, plasmids, and cell transfection

U87MG and SNB19 cells were transfected with either the siRNAs targeting TRAF4 (si-TRAF4\#1 or siTRAF4\#2) or the scrambled control oligonucleotide (Scr; RiboBio, Guangzhou, China; Supplementary Table 3) using X-tremeGENE siRNA Transfection Reagent (Roche, 
Table 1 Correlation between miR-29a/b/c and TRAF4 expressions and clinicopathological characteristics of $187 \mathrm{glioma}$ patients

Feature

Mutant type (IDH1 73

R132H)

Wild type (IDH1/2) 114

Gender

Male

Female

Age

Age $<50$

81

Age $\geq 50$

KPS

KPS $<90$

$\mathrm{KPS} \geq 90$

WHO grade

$$
\text { II }
$$

III

IV

\section{Ki-67 LI}

$\mathrm{LI}<29.01$

Predominant side

Left

Right

Middle

Predominant location

Frontal lobe 103

Frontal lobe $\quad 103$

Temporal lobe

Parietal lobe

106

117

70

40

40

94
Number of MiR-29a LI $X^{2} / P$-value

cases

High Low

MiR-29b LI $x^{2} / P$-value

High Low

MiR-29c LI $x^{2} / P$-value

High Low

TRAF4 LI $\quad X^{2} /$ P-value

High Low

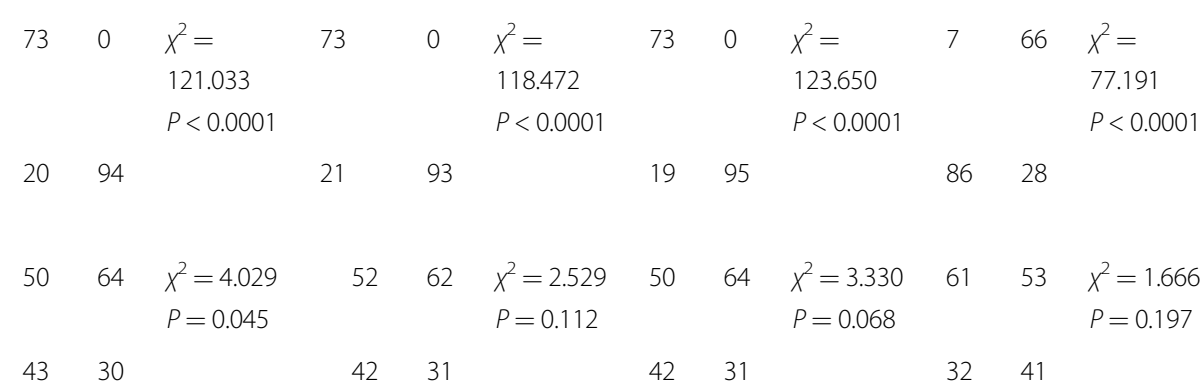

$60 \quad 21 \quad x^{2}=33.868$

$P<0.0001$

62

19

$x^{2}=39.464$

$P<0.0001$

61

20

$x^{2}=38.979$

$P<0.0001$

$22 \quad 59 \quad x^{2}=$

29.123

$P<0.0001$

$33 \quad 73$

$32 \quad 74$

$31 \quad 75$

$71 \quad 35$

$50 \quad 67 \quad x^{2}=6.122$

$50 \quad 67 \quad x^{2}=7.094$

$P=0.013$

$P=0.008$

$49 \quad 68 \quad x^{2}=6.696$

$P=0.010$

$63 \quad 54 \quad x^{2}=2.116$

$43 \quad 27$

$44 \quad 26$

$43 \quad 27$

$P=0.146$

$x^{2}=141.316$
$P<0.0001$

$40 \quad 0 \quad x^{2}=$

138.326

$P<0.0001$

$40 \quad 0$

$x^{2}=$

144.372

$P<0.0001$

$30 \quad 40$

$40 \quad 0$

$40 \quad 0$

$14 \quad 93$

$40 \quad 0$

1295

$81 \quad 26$

$13 \quad 94$

$\begin{aligned} 91 \quad 2 \quad & x^{2}=171.342 \\ & P<0.0001\end{aligned}$

93

0

$x^{2}=$

183.042

$91 \quad 2$

$P<0.0001$

175.212

$13 \quad 80 \quad x^{2}=$

$P<0.0001$

94.607

$P<0.0001$

292

193

93

$80 \quad 14$ $P<0.0001$

$44 \quad 51 \quad x^{2}=1.201$

43

$P=0.549$

$x^{2}=2.109$

$P=0.348$

$43 \quad 52 \quad x^{2}=1.495$

$P=0.473$

$50 \quad 45 \quad x^{2}=0.686$

$44 \quad 40$

$46 \quad 38$

$44 \quad 40$

$P=0.709$

53

53

53

$39 \quad 45$

44 
Table 1 continued

\begin{tabular}{|c|c|c|c|c|c|c|c|c|c|}
\hline Occipital lobe & 8 & 2 & 6 & 2 & 6 & 2 & 6 & 7 & 1 \\
\hline Insular lobe & 1 & 1 & 0 & 1 & 0 & 1 & 0 & 0 & 1 \\
\hline Cerebellum & 4 & 3 & 1 & 3 & 1 & 3 & 1 & 1 & 3 \\
\hline CPA & 1 & 0 & 1 & 0 & 1 & 0 & 1 & 0 & 1 \\
\hline Third ventricle & 1 & 1 & 0 & 1 & 0 & 1 & 0 & 1 & 0 \\
\hline
\end{tabular}

LI labeling index, KPS Karnofsky performance score

Indianapolis, IN, USA). The TRAF4 expression plasmid pTRAF4 was constructed by GeneCopoeia (Rockville, MD, USA) and was validated by DNA sequencing. Plasmid transfection was performed using X-tremeGENE HP DNA Transfection Reagent (Roche, Indianapolis, IN, USA).

\section{Quantitative RT-PCR}

qRT-PCR was performed as previously described ${ }^{7}$. The Bulge-Loop $^{\text {TM }}$ miRNA qRT-PCR primer sets for hsa-miR29a/b/c were purchased from Ribobio (Guangzhou, China). Total RNA $(1 \mu \mathrm{g})$ was reverse-transcribed with either the miRNA-specific primers or an Oligo dT primer using a Reverse Transcription System Kit (Promega, Fitchburg, WI, USA). Subsequently, qRT-PCR was performed with a GoTaq qPCR Master Mix Kit (Promega, Fitchburg, WI, USA) to quantify the miRNA and mRNA levels using U6 and GAPDH as the internal controls, respectively. The specific primers used for TRAF4 mRNA detection are listed in Supplementary Table 4. The fold changes of the miRNA and mRNA levels were calculated by the $2^{-\Delta \Delta C t}$ method.

\section{Flow cytometry assay}

For the apoptosis assay, cells were labeled with an Annexin V-APC/PI Apoptosis Detection Kit (KeyGEN, Nanjing, China) according to the manufacturer's instructions. For cell cycle analysis, cells were harvested, washed with ice-cold PBS, fixed in $70 \%$ ethanol at $4{ }^{\circ} \mathrm{C}$ overnight, and labeled with PI (Solarbio, Beijing, China) in the presence of RNase A (Solarbio, Beijing, China) at $37^{\circ}$ $\mathrm{C}$ for $30 \mathrm{~min}$. Then, the cells were read on an Accuri C6/ FACSCalibur Flow Cytometer (BD Biosciences, Franklin Lakes, NJ, USA), and the data were processed with FlowJo/ModFit LT software.

\section{Cell apoptosis assays}

Alkaline single cell gel electrophoresis (SCGE) detection was performed as previously described ${ }^{7}$. Briefly, cells $(1 \times$ $10^{4}$ ) were harvested and suspended in $0.5 \%(\mathrm{w} / \mathrm{v})$ low melting agarose at $37^{\circ} \mathrm{C}$, and then the suspensions were layered on slides pre-coated with a layer of $0.6 \%(\mathrm{w} / \mathrm{v})$ normal-melting agarose. The slides were then immersed in cold lysing solution for $2 \mathrm{~h}$ at $4{ }^{\circ} \mathrm{C}$. After electrophoresis, the slides were stained with ethidium bromide, and the images were acquired using a DM6000B fluorescence microscope (Leica, Wetzlar, Germany). Apoptotic indexes $[\mathrm{AIs}(\%)]$ were calculated as apoptotic cell numbers/total cell numbers $\times 100 \%$. Caspase $3 / 7$ activity was measured with a Caspase-Glo ${ }^{\circledR}$ 3/7 Assay Kit (Promega, Fitchburg, WI, USA) according to the manufacturer's instructions. Luminescence was measured after a 30-min incubation with Caspase-Glo ${ }^{\circledR}$ 3/7 Reagent in a Synergy $^{\text {TM }} 2$ Multi-Mode Microplate Reader (BioTek Instruments, Winooski, VT, USA).

\section{Cell proliferation assays}

A 5-ethynyl-2'-deoxyuridine (EdU) incorporation assay was performed with a Cell-Light ${ }^{\mathrm{TM}}$ EdU Apollo ${ }^{\circledR} 567$ In Vitro Imaging kit (RiboBio, Guangzhou, China). Cells $\left(1.5 \times 10^{3}\right.$ cells/well $)$ were seeded in 96 -well plates and incubated with EdU $(50 \mu \mathrm{M})$ for $2 \mathrm{~h}$. The cells were then fixed in $4 \%$ paraformaldehyde and stained with Apollo and Hoechst 33342. Cells in five randomly selected microscopic fields were counted and the images were captured with a fluorescence microscope (Olympus, Tokyo, Japan). EdU-positive rates (\%) were calculated as EdU-positive cell numbers/Hoechst-stained cell numbers $\times 100 \%$. For the MTS assay, cells $\left(1.5 \times 10^{3}\right.$ cells/well $)$ were seeded in 96-well plates and incubated for $24-120 \mathrm{~h}$. Before measurements were obtained, the supernatants were replaced with fresh medium containing 3-(4,5dimethylthiazol-2-yl)-5-(3-carboxymethoxyphenyl)-2-(4sulfophenyl)-2H-tetrazolium (MTS, Promega, Fitchburg, WI, USA), and the cells were cultured for another $2.5 \mathrm{~h}$. Then the absorbance at $490 \mathrm{~nm}$ was measured with a Synergy $^{\text {тм }} 2$ Multi-Mode Microplate Reader (BioTek Instruments, Winooski, VT, USA). Data at each interval during a 24-h period were documented.

\section{Target prediction and dual-luciferase reporter assay}

The candidate targets of $\mathrm{miR}-29 \mathrm{a} / \mathrm{b} / \mathrm{c}$ were predicted by Targetscan, PicTar, and MicroCosm. The DNA fragment corresponding to the $3^{\prime}$ - untranslated region of TRAF4 mRNA (TRAF4-3'-UTR-WT) was amplified by RT-PCR and inserted into the pEZX-MT01 vector (GeneCopoeia, Rockville, MD, USA). Then, target region 1 or 2 of $\mathrm{miR}-29 \mathrm{a} / \mathrm{b} / \mathrm{c}$ was deleted individually 


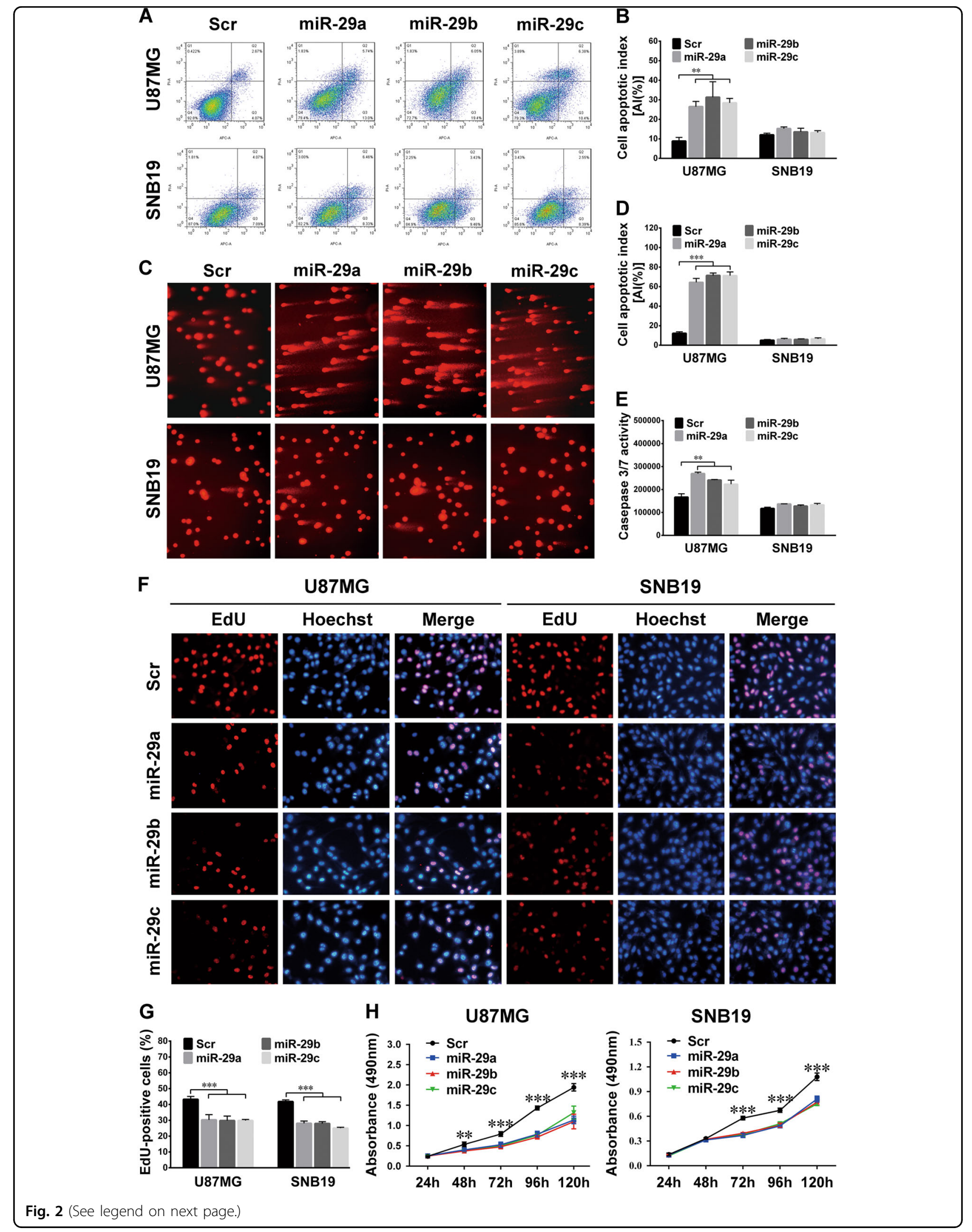


(see figure on previous page)

Fig. 2 miR-29a/b/c induce apoptosis and inhibit the proliferation of glioma cells. a, b The representative FCM cell apoptosis analysis results (a) and the apoptotic indexes (Als\%; b). The Als\% were compared among the miR-29a/b/c-overexpressing sub-cell lines and the control sub-cell lines (Scr) of U87MG and SNB19 cells. c, d Representative results of SCGE (c), Als were calculated as the percent ratio of long-tailed cells to total cells (d) and compared as described in $\mathbf{b}$. e Results of the caspase $3 / 7$ activity analysis of the cells as indicated. $\mathbf{f}, \mathbf{g}$ Representative images of the EdU assay (f) and the quantification results $(\mathbf{g})$. The EdU-positive and Hoechst-stained cells were counted in the microscopic fields at $\times 200$. $\mathbf{h}$ Growth curves of the above sub-cell lines. The absorbances at $490 \mathrm{~nm}$ at the indicated time points were measured by MTS assays. All the experiments were performed at least in triplicate and the data in $\mathbf{b}, \mathbf{d}, \mathbf{e}, \mathbf{g}$, and $\mathbf{h}$ are presented as the mean $\pm \mathrm{SD}$. ${ }^{* *} P<0.01$; ${ }^{* *} P<0.001$

(TRAF4-3'-UTR-MT1 and TRAF4-3'-UTR-MT2) by site-directed mutagenesis PCR using the primers listed in Supplementary Table 5. The recombinant luciferase reporter plasmids containing TRAF4-3'-UTR-WT, TRAF4-3'-UTR-MT1, and TRAF4-3'-UTR-MT2 were named p-WT, p-MT1, and p-MT2, respectively. Firefly and Renilla luciferase activities were detected with a Dual-Luciferase Reporter Assay System (Promega, Fitchburg, WI, USA) according to the manufacturer's instructions in a Synergy ${ }^{\mathrm{Tm}} 2$ Multi-Mode Microplate Reader (BioTek Instruments, Winooski, VT, USA). Firefly luciferase activity was adjusted according to that of Renilla luciferase to normalize the differences in transfection efficiencies.

\section{Western blotting}

Western blotting was performed as described previously ${ }^{6}$. Rabbit anti-human TRAF4 antibody and mouse anti-human MDM2 and p53 antibodies were purchased from Santa Cruz Biotechnology (Santa Cruz, CA, USA). Rabbit anti-human pAKT (phospho S473), AKT, p-MDM2 (Phospho Ser166), Bax, p-GSK-3 $\beta$ (phospho S9), GSK-3 $\beta$, cyclin D1, and c-Myc antibodies were purchased from CST (Boston, MA, USA). Mouse anti-human $\beta$-actin and GAPDH antibodies were purchased from Boster (Wuhan, China).

\section{Statistical analysis}

Data are presented as the means \pm standard deviation (SD). Corresponding data in this study were analyzed by one-way ANOVA, $\chi^{2}$ test, Pearson correlation analysis, Kaplan-Meier analysis, log-rank test, and Student's $t$-test. In the survival analysis, the median of each glioma cohort was used as the cutoff for risk stratification. All the analyses were performed using the SPSS 21.0 software package (IBM, Chicago, IL, USA), and statistical significance was assigned at $P<0.05(* / \boldsymbol{\Delta}), P<0.01(* * / \Delta \boldsymbol{\Delta})$, or $P<$ $0.001(* * * \mathbf{\Delta} \boldsymbol{\Delta})$. All the experiments on cell lines were performed at least three times with triplicate samples.

\section{Results}

$\mathrm{miR}-29 \mathrm{a} / \mathrm{b} / \mathrm{c}$ are decreased in human gliomas and correlate with poor prognosis

To reveal the relationship among miR-29a/b/c levels and glioma grade, tumor cell proliferation and patient prognoses, we detected the endogenous levels of miR-29a/ $\mathrm{b} / \mathrm{c}$ and Ki-67 in 187 human glioma specimens and 20 nontumoral control brain tissues. We found that miR$29 \mathrm{a} / \mathrm{b} / \mathrm{c}$ mainly located at the perinuclear zone and their levels were significantly lower in gliomas than in control brain tissues $(P<0.001)$ and that the levels declined progressively as the glioma grade increased $(P<0.001$; Fig. 1a, b). A Pearson analysis showed that all the labeling indexes of the three miR-29s were inversely correlated with the proliferation index (Ki-67 LI; miR-29a: $r=-0.798$; miR29b: $r=-0.805$; miR-29c: $r=-0.818 ; P<0.0001$; Fig. 1c and Supplementary Fig. 1a, b). Importantly, the decreases in $\mathrm{miR}-29 \mathrm{a} / \mathrm{b} / \mathrm{c}$ levels were significantly associated with the wild-type $I D H 1 / 2$ gene, older age, advanced tumor grade, and higher Ki-67 LI $(P<0.0001$; Table 1). The qRT-PCR results verified that the miR-29a/b/c expression levels were also significantly reduced in six human glioblastoma cell lines compared with the human astrocyte cell line UC2 (Supplementary Fig. 2a, b). Kaplan-Meier analyses demonstrated that the patients with relatively higher miR-29a/b/c levels exhibited longer DFS $(P<$ $0.0001)$ and $\mathrm{OS}(P<0.0001$; Fig. 1d), even within the cohort of patients with the same tumor grade (Fig. 1e and Supplementary Fig. 3a, b), identical IDH status (Fig. 1f, g), similar age, and KPS (Supplementary Fig. 4a-d). These data demonstrate the inverse association of $\mathrm{miR}-29 \mathrm{a} / \mathrm{b} / \mathrm{c}$ levels with glioma grade and tumor cell proliferation, and imply their potential value as prognostic biomarkers for glioma patients.

\section{$\mathrm{miR}-29 \mathrm{a} / \mathrm{b} / \mathrm{c}$ induce apoptosis and suppress the proliferation of glioma cells}

To investigate the effects of miR-29a/b/c on tumor cell apoptosis and proliferation, we established sub-cell lines that stably overexpressed miR-29a/b/c (termed miR-29a, miR-29b, and miR-29c) or the control (Scr). The expression efficiencies of the miRNAs were verified by qRT-PCR $(P<0.01-0.001$; Supplementary Fig. 5a, b). For U87MG with the wild-type TP53 gene ${ }^{26,27}$, the apoptosis indexes (AIs) of the miR-29a/b/c sub-cell lines were significantly higher than those of the Scr, as indicated by the results of flow cytometry (FCM) $(P<0.01$; Fig. 2a, b), SCGE $(P<0.001$; Fig. $2 \mathrm{c}, \mathrm{d})$, and the caspase $3 / 7$ activity assay $(P<0.01$; Fig. $2 \mathrm{e})$. These results were also obtained 


\section{A}

3'-UTR of TRAF4 mRNA

\begin{tabular}{|c|c|c|}
\hline & Target region & \\
\hline $\begin{array}{l}\text { TRAF4-3'-UTR 161-167bp } \\
\text { (Target region 1) }\end{array}$ & $5^{\prime} \ldots$... UCAGGUGCCUCCAAL & UUGGUGCUU...3 \\
\hline sa-miR-29a & 3' AUUGGCUAAAGUCl & UACCACGAU : \\
\hline sa-miR-29b & 3' UUGUGACUAAAGUUL & IUACCACGAU : \\
\hline sa-miR-29c & 3' AUUGGCUAAAGUU & UACCACGAU : \\
\hline $\begin{array}{l}\text { TRAF4-3'-UTR 310-316bp } \\
\text { (Target region 2) }\end{array}$ & $5^{\prime}, \ldots$ UGAGGUGCCUGCU & $\begin{array}{l}\text { AGGUGCUAU... } \\
11111111\end{array}$ \\
\hline nsa-miR-29a & 3' AUUGGCUAAAGUCU & UACCACGAU 5 \\
\hline sa-miR-29b & 3' UUGUGACUAAAGU & UACCACGAU \\
\hline sa-miR-29c & AUUGGCUA & CCACGAU \\
\hline
\end{tabular}

B

The sequence of TRAF4-3'-UTR-WT ( $p$-WT)

Target region $1 \quad$ Target region 2

5'..UCAGGUGCCUCCAAU UGGUGCU ..UGAGGUGCCUGCUCAGGUGCUA ...'

The sequence of TRAF4-3'-UTR-MT1 (p-MT1)

Target region 1

Target region 2

5'..UCAGGUGCCUCCAAU UGGUGCUU..UGAGGUGCCUGCUCA GGUGCUAU...3'

The sequence of TRAF4-3'-UTR-MT2 (p-MT2)

Target region 1

Target region 2

5'..UCAGGUGCCUCCAAUUGGUGCU .UGAGGUGCCUGCUCAGGUGGUAU...3'

E

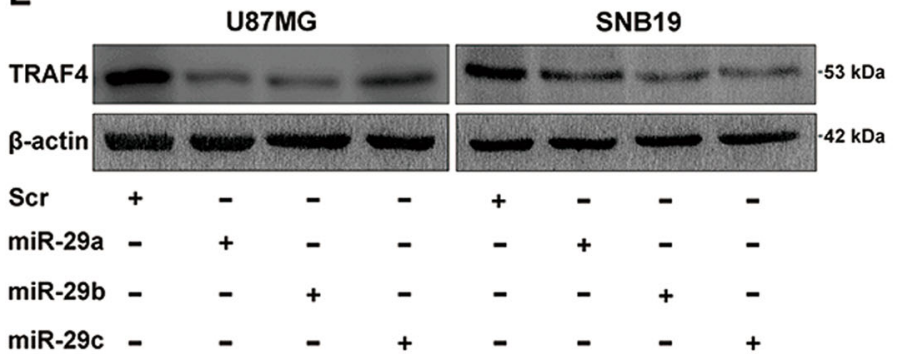

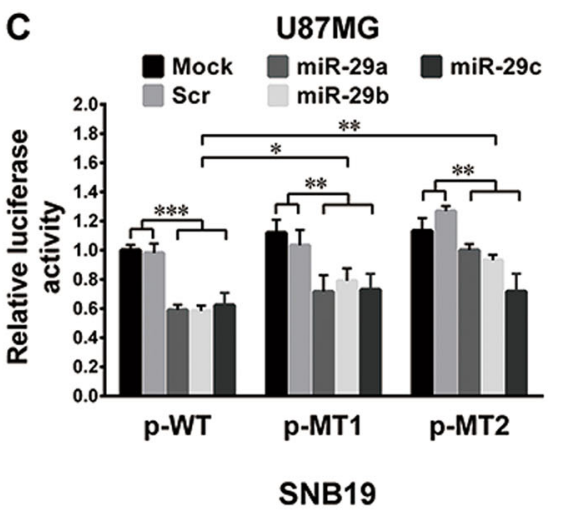
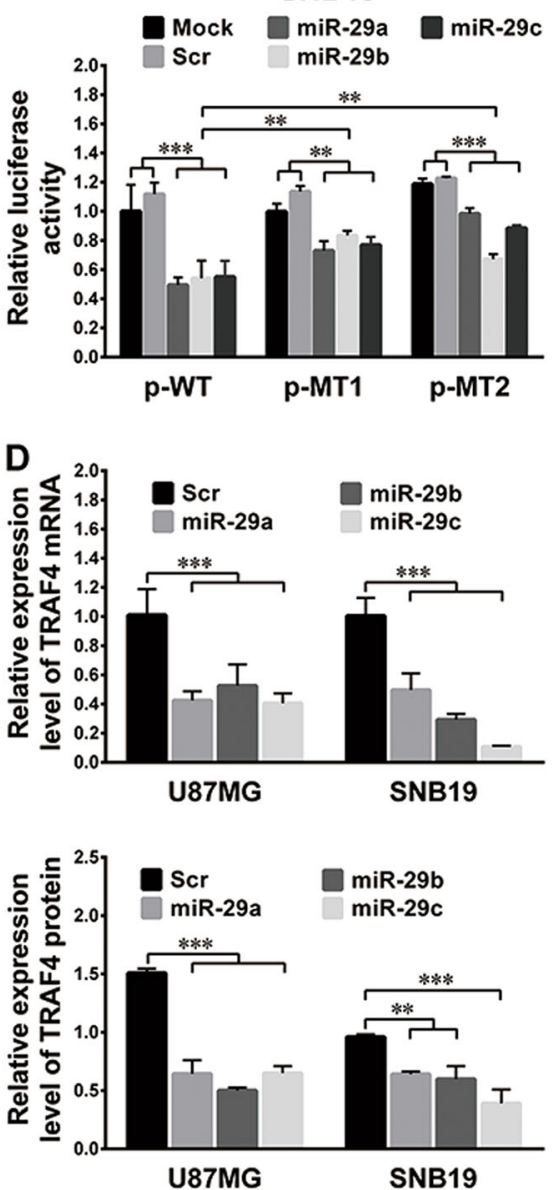

Fig. 3 miR-29a/b/c directly target TRAF4 in glioma cells. a miR-29a/b/c target regions in the $3^{\prime}-U T R$ of TRAF4 mRNA as predicted by the bioinformatics analysis. $\mathbf{b}$ Schematic illustration of the transcripts of the recombinant luciferase reporter plasmids used in the luciferase assays. The transcript of p-WT (TRAF4-3'-UTR-WT) contains all the target regions for miR-29a/b/c. In the transcripts of p-MT1 and p-MT2 (TRAF4-3'-UTR-MT1 and TRAF4-3'-UTR-MT2), target region 1 or target region 2 was deleted, respectively. c Dual-luciferase reporter assays results. U87MG and SNB19 cell lines (Mock) and their Scr and miR-29a/b/c sub-cell lines were transfected with the luciferase reporter plasmid as indicated. d, e miR-29a/b/c effectively suppressed the expression of TRAF4 mRNA and protein in the miR-29a/b/c sub-cell lines of U87MG and SNB19 cells. All the experiments were performed at least in triplicate and the data in c-e are presented as the mean \pm SD. ${ }^{*} P<0.05 ;{ }^{* *} P<0.01 ;{ }^{* *} P<0.001$

in DBTRG-05MG cells in which the TP53 gene was also wild type ${ }^{28}(P<0.001$; Supplementary Fig. 5c, d). However, in SNB19 and U251 cells, which bear a mutation at amino acid 273 of $\mathrm{p} 53^{27,29,30}$, no obvious difference in apoptosis was observed between the control and the miR29a/b/c-overexpressing groups (Fig. 2a-e and Supplementary Fig. 5c, d). The EdU and MTS assays showed that $\mathrm{miR}-29 \mathrm{a} / \mathrm{b} / \mathrm{c}$ could effectively inhibit the proliferation of 


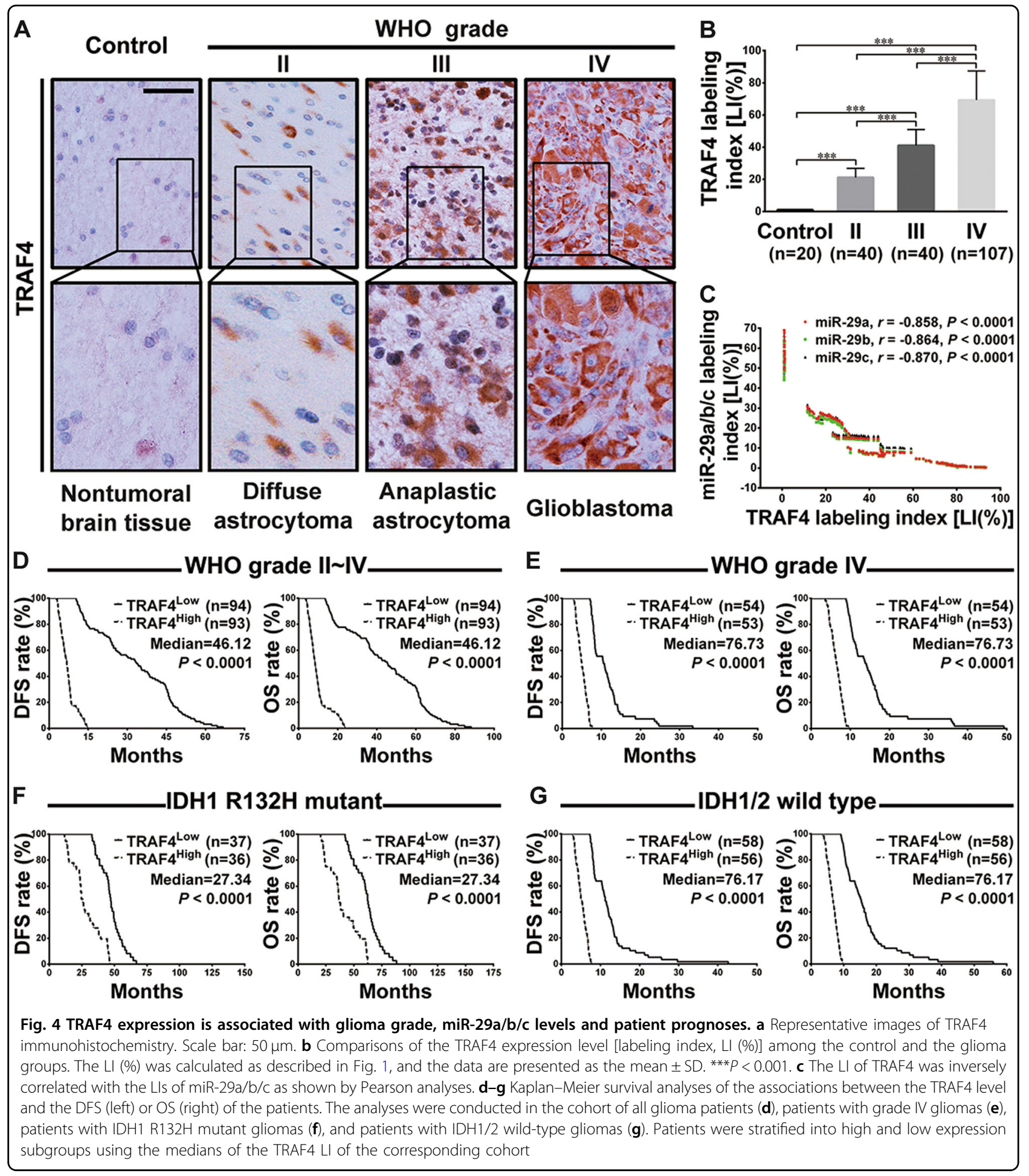

U87MG and SNB19 cells $(P<0.01-0.001$; Fig. $2 \mathrm{f}-\mathrm{h})$. Combining with the above findings that miR-29a/b/c LIs were inversely correlated with the Ki-67 LI, our results suggest that, as pro-apoptotic and antiproliferative miRNAs, miR-29a/b/c are effective glioma suppressors.
TRAF4 is a direct target of miR-29a/b/c in human glioma cells

For the bioinformatics prediction, two regions in the 3'UTR of TRAF4 mRNA were predicted to be the putative targets of miR-29a/b/c (Fig. 3a). The silencing effects of the miRNAs were confirmed in U87MG and SNB19 cells 


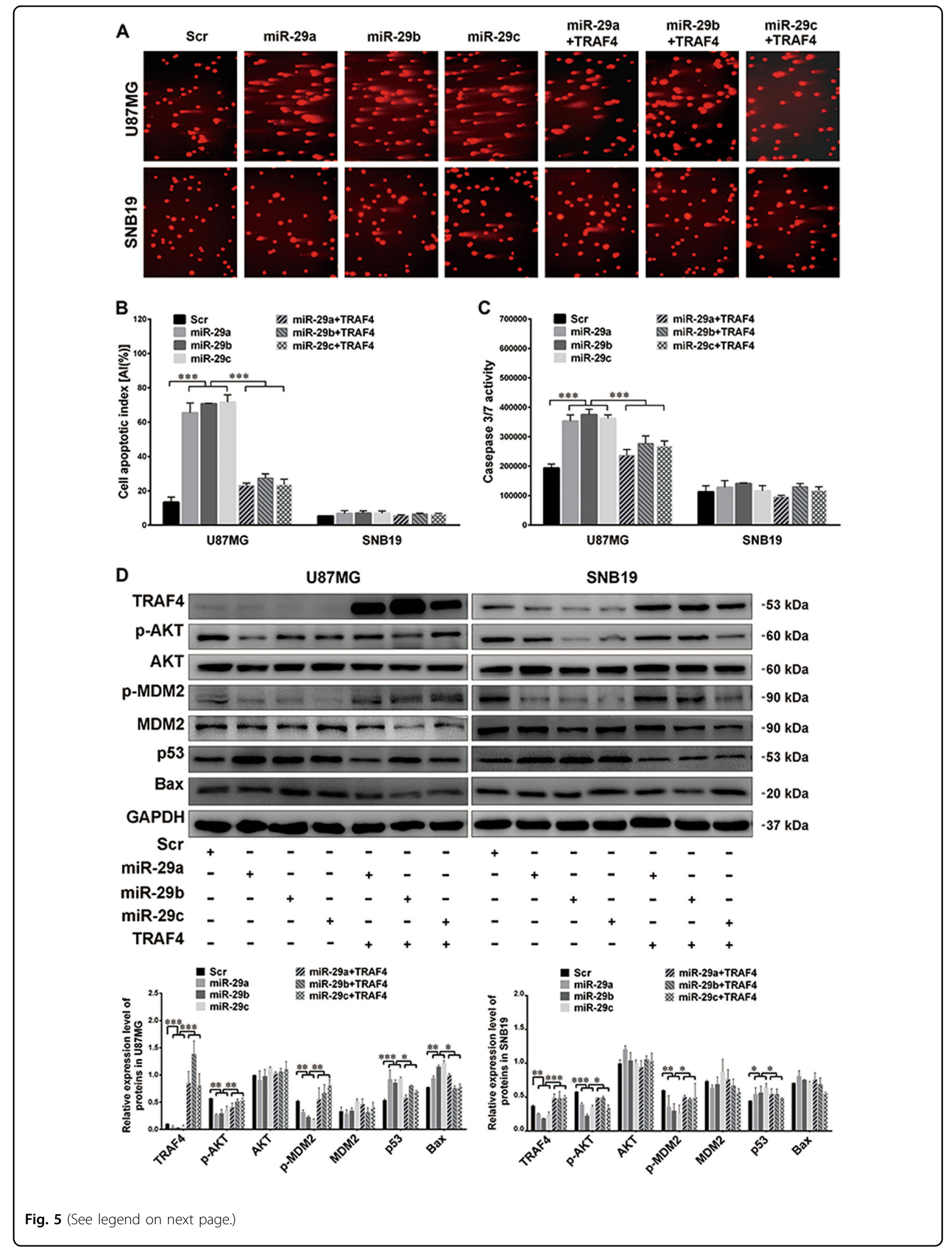


(see figure on previous page)

Fig. 5 miR-29a/b/c promote glioma cell apoptosis through the TRAF4/AKT/MDM2/p53 pathway. a, b Representative images of SCGE (a) and the comparison of Als $(\mathbf{b})$. Apoptosis was detected in the Scr and the miR-29a/b/c sub-cell lines of U87MG and SNB19 cells, and the miR-29a/b/coverexpressing cells transfected with the TRAF4 expression plasmid. c Results of the caspase 3/7 activity assay of the cells as indicated. $\mathbf{d}$ The levels of TRAF4, p-AKT, AKT, p-MDM2, MDM2, p53, and Bax in the extracts of the above cells were detected by western blot using GAPDH as the internal control. All the experiments were performed at least in triplicate and the data in $\mathbf{b}$ - $\mathbf{d}$ are presented as the mean $\pm \mathrm{SD}$. ${ }^{*} P<0.05 ;{ }^{* *} P<0.01 ;{ }^{* * *} P<$ 0.001

by dual-luciferase reporter assays $(P<0.05-0.001$; Fig. 3b, c), which indicated that TRAF4 was a direct target of miR$29 \mathrm{a} / \mathrm{b} / \mathrm{c}$ in glioma cells. In addition, the qRT-PCR and western blot results further verified that $\mathrm{miR}-29 \mathrm{a} / \mathrm{b} / \mathrm{c}$ significantly decreased the mRNA and protein levels of TRAF4 $(P<0.01-0.001$; Fig. $3 \mathrm{~d}$, e). These data demonstrate that $\mathrm{miR}-29 \mathrm{a} / \mathrm{b} / \mathrm{c}$ bind directly to the target regions in the 3'-UTR of TRAF4 mRNA and inhibit the expression of TRAF4 via the induction of the degradation of its mRNA.

\section{TRAF4 overexpression is correlated with $\mathrm{miR}-29 \mathrm{a} / \mathrm{b} / \mathrm{c}$ down-regulation and predicts a poorer prognosis}

We then sought to detect the endogenous TRAF4 levels in the above glioma specimens and control brain tissues. The IHC results showed that the TRAF4 level was higher in gliomas than in the controls $(P<0.001)$ and continued to increase as the glioma grade increased $(P<0.001$; Fig. 4a, b). This result was further verified by the four published datasets from the Oncomine database $(P<$ 0.0001; Supplementary Fig. 6d). Moreover, in our glioma specimens, the TRAF4 level was negatively correlated with the $\mathrm{miR}-29 \mathrm{a} / \mathrm{b} / \mathrm{c}$ levels (miR-29a: $r=-0.858$; miR29b: $r=-0.864$; miR-29c: $r=-0.870 ; P<0.0001$; Fig. 4c). Accordingly, the increase in the TRAF4 level was positively associated with the wild-type $I D H 1 / 2$ gene, older age, advanced tumor grade, and higher Ki-67 LI $(P<$ 0.0001; Table 1 and Supplementary Fig. 1c). Kaplan-Meier analyses demonstrated that a higher TRAF4 level predicted a shorter DFS $(P<0.0001)$ and OS $(P<0.0001$; Fig. 4 d) of our glioma patients. Even within the cohort with similar clinicopathologic features, including tumor grade (Fig. 4e and Supplementary Fig. 6a), IDH gene status (Fig. 4f, g), age, and KPS scores (Supplementary Fig. 6b, c), stratification of the patients according to TRAF4 level could still perfectly reflect the differences in outcomes, i.e., the higher the TRAF4 level, the shorter the OS and DFS of the patients (DFS: $P<$ 0.0001; OS: $P<0.0001$ ). The prognostic value of TRAF4 in gliomas was further validated by data from TCGA database (OS: $P<0.0001$; DFS: $P<0.01$; Supplementary Fig. 6e). These data indicate that the abnormal decrease in $\mathrm{miR}-29 \mathrm{a} / \mathrm{b} / \mathrm{c}$ levels are important causes of TRAF4 overexpression in gliomas and imply the potential value of TRAF4 in the prognosis of patients with gliomas.
miR-29a/b/c promote glioma cell apoptosis by blocking the TRAF4/AKT/MDM2/p53 pathway

To ascertain whether miR-29a/b/c induce cell apoptosis by silencing TRAF4, we transfected the miR-29a/b/coverexpressing sub-cell lines with the TRAF4-expressing plasmid. The apoptosis detection results showed that although $\mathrm{miR}-29 \mathrm{a} / \mathrm{b} / \mathrm{c}$ dramatically promoted apoptosis in U87MG and DBTRG-05MG cells, their effects were potentially abrogated by exogenous TRAF4 $(P<0.001$; Fig. 5a-c and Supplementary Fig. 7a, b). However, the apoptotic levels of SNB19 remained constant during miR$29 \mathrm{a} / \mathrm{b} / \mathrm{c}$ overexpression and TRAF4 restoration $(P>0.05$; Fig. $5 \mathrm{a}-\mathrm{c})$. To further understand the intracellular signaling network, we focused on p53 and the AKT/MDM2 pathway. Western blotting confirmed that miR-29a/b/c significantly reduced the levels of phosphorylated AKT (p-AKT) and phosphorylated MDM2 (p-MDM2) and increased the levels of p53 in U87MG and SNB19 cells, while TRAF4 overexpression effectively reversed the changes mentioned above $(P<0.05-0.001 ;$ Fig. $5 \mathrm{~d})$. However, the total AKT and MDM2 levels were exempted from the impacts of both miR-29a/b/c and TRAF4. Most importantly, the expression of Bax was significantly increased by miR-29a/b/c, and returned when TRAF4 was overexpressed in U87MG cells with the wild-type TP53 gene $(P<0.05-0.01$; Fig. 5 d), but not in SNB19 with mutant TP53 (Fig. 5d). Overall, these results demonstrate that $\mathrm{miR}-29 \mathrm{a} / \mathrm{b} / \mathrm{c}$ markedly induce the apoptosis of glioma cells in a p53-dependent manner.

\section{miR-29a/b/c restrain glioma cell proliferation through the TRAF4/AKT/GSK-3 $\beta$ pathway}

To determine whether TRAF4 silencing mediates the adverse effects of miR-29a/b/c on glioma cell proliferation, we transfected the mixture of the miR-29a/ b/c-overexpressing sub-cell lines (miR-29s) with the TRAF4-expressing plasmid (miR-29s + TRAF4). The FCM (Fig. 6a), EdU (Fig. 6b, c), and MTS (Fig. 6d) assay results demonstrated that miR-29s dramatically suppressed G1/S-phase transition and tumor cell proliferation, while ectopic TRAF4 efficaciously compromised these effects $(P<0.05-0.001)$. Additionally, transient knockdown of endogenous TRAF4 with specific siRNAs $(P<0.001 ; \quad$ Supplementary Fig. 7c, d) perfectly simulated the suppressive effects 


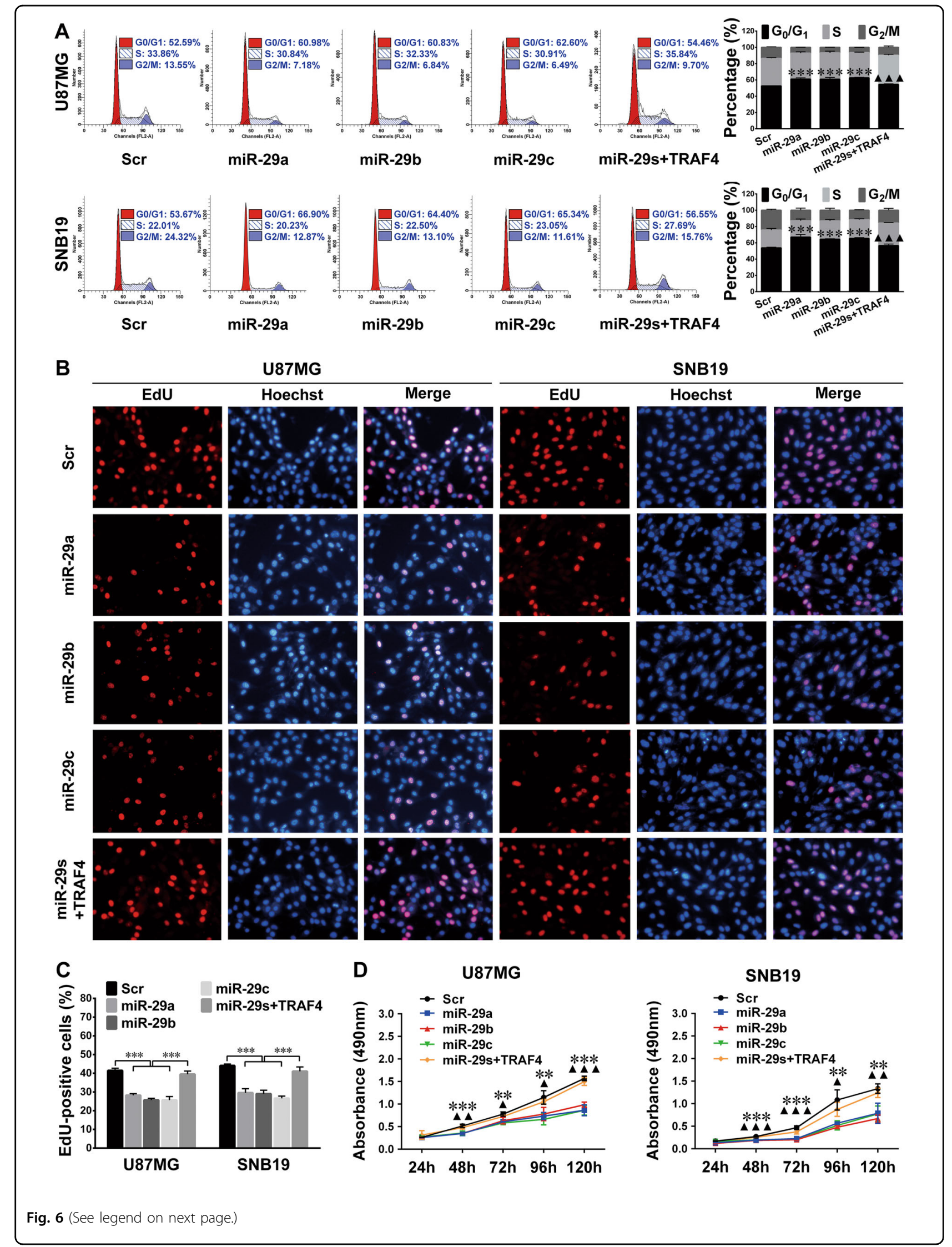


(see figure on previous page)

Fig. 6 miR-29a/b/c block cell cycle progression and glioma cell proliferation by targeting TRAF4. a The representative FCM cell cycle results (left) and the percentages of cells in each phase (right). The percentages of cells in each phase were compared among the Scr and miR-29a/b/c subcell lines and the mixture of miR-29a/b/c-overexpressing cells transfected with the TRAF4 expression plasmid (miR-29s + TRAF4). b, c EdU assay results $(\mathbf{b})$ and quantitative analyses $\mathbf{c}$ of the aforementioned cells. The EdU-positive and Hoechst-stained cells were counted as described in Fig. 2. $\mathbf{d}$ Growth curves of the indicated cells drawn with absorbances obtained in MTS assays. All the experiments were performed at least in triplicate and the data in $\mathbf{a}, \mathbf{c}, \mathbf{d}$ are presented as the mean \pm SD. $\mathbf{\Delta}_{P<0.05 ;} \mathbf{\Delta \Lambda}_{* * P}^{*}<0.01 ; \mathbf{\Delta \Lambda \mathbf { \Lambda }}{ }_{* * *}^{*} p<0.001$. The miR-29a/b/c groups compared with the Scr group $\left(^{*}\right)$ or the miR-29s + TRAF4 group $(\boldsymbol{\Delta})$ in $\mathbf{a}, \mathbf{d}$

of $\mathrm{miR}-29 \mathrm{a} / \mathrm{b} / \mathrm{c}$ on cell cycle progression and glioma cell proliferation, which were also partially reversed by TRAF4 overexpression $(P<0.01-0.001$; Fig. $7 \mathrm{a}-\mathrm{d})$.

To further explore the pathway through which miR29a/b/c suppress glioma cell proliferation, we focused on the crucial effectors downstream of TRAF4, including AKT, GSK-3 $\beta$, c-Myc, and the G1/S-phase checkpoint regulator cyclin D1. As shown in Fig. 8a, b, the levels of

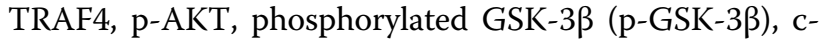
Myc, and cyclin D1 were significantly reduced in U87MG and SNB19 cells that overexpressed miR-29a/b/c or that were transfected with TRAF4 siRNAs $(P<0.05-0.001)$. In contrast, the total AKT and GSK-3 $\beta$ levels remained constant among all the groups. Moreover, TRAF4 overexpression reversed the above-mentioned changes in protein levels induced by $\mathrm{miR}-29 \mathrm{a} / \mathrm{b} / \mathrm{c}$ restoration and TRAF4 knockdown $(P<0.05-0.001)$. These results indicate that miR-29a/b/c inhibit G1/S-phase transition and glioma cell proliferation by direct targeting of the TRAF4/ AKT/GSK-3 $\beta$ pathway and the suppression of c-Myc and cyclin D1 expression (Fig. 8c).

\section{Discussion}

The miR-29 family members have been recognized as important tumor suppressors in various malignant tumors $^{13-17,20}$. However, their anti-tumor roles and clinical relevance remain to be investigated in a large cohort of glioma patients. In the present study, we demonstrated that $\mathrm{miR}-29 \mathrm{a} / \mathrm{b} / \mathrm{c}$ effectively induced apoptosis and inhibited the proliferation of glioma cells by targeting TRAF4, and thereby we validated the miR- 29 members as important glioma suppressors. We also demonstrated that these suppressive miRNAs were expressed at low levels in gliomas, especially in high-grade gliomas and that their levels were closely associated with patient outcomes. These findings not only enrich our knowledge of the crucial molecular events during glioma formation, but they also imply the usefulness of these miRNAs as prognostic biomarkers for glioma patients.

A comprehensive study of miRNAs and their regulatory networks is a prerequisite for the scientific evaluation of their practical values in the molecular subclassification, diagnosis, and prognosis of gliomas ${ }^{6-9}$. In this study, we verified that the expression levels of $\mathrm{miR}-29 \mathrm{a} / \mathrm{b} / \mathrm{c}$ and TRAF4 were closely correlated with tumor grade and proliferation index, which suggests that they can be used as accessory indicators for glioma grading. A survival analysis showed that even within the current frame of prognostic evaluation (including pathological grade, IDH status, age and KPS), the expression levels of miR-29a/b/c and TRAF4 could still provide us with additional reliable predictive information on patient outcomes and could improve the accuracy of the prognostic assessment. In our pathologic analysis, we also found that the Ki-67 index, a widely accepted scale for proliferation, was not only increased as the glioma grade increased but also inversely correlated with miR-29a/b/c levels and was positively correlated with the TRAF4 level. This strongly indicates that $\mathrm{miR}-29 \mathrm{a} / \mathrm{b} / \mathrm{c}$ and TRAF4 are important regulators of glioma cell proliferation. Moreover, the inverse correlations between the levels of miR-29a/b/c and TRAF4 imply that the abnormal decrease in miR-29s is responsible for the overexpression of TRAF4 in gliomas. This conclusion was further supported by the luciferase assay results which confirmed that TRAF4 was a natural target of miR$29 \mathrm{a} / \mathrm{b} / \mathrm{c}$ in glioma cells.

Apoptotic inhibition and vigorous proliferation of tumor cells are not only the hallmarks of malignant gliomas, but they are also important cause of their rapid growth $^{3,31}$. Our in vitro results showed that all the miR-29 members efficiently inhibited the proliferation of glioma cells and that they also induced apoptosis in certain glioma cell lines such as U87MG and DBTRG-05MG. Furthermore, since the above-mentioned anti-glioma effects of miR-29a/b/c could be simulated perfectly by the specific siRNAs of TRAF4 and could be partially reversed by TRAF4 overexpression, we conclude that $\mathrm{miR}-29 \mathrm{a} / \mathrm{b} / \mathrm{c}$ suppress glioma growth at least partly by silencing TRAF4. These results highlight the importance of miR$29 \mathrm{a} / \mathrm{b} / \mathrm{c}$ as tumor suppressors to combat malignant gliomas.

TRAF4 has recently been found to promote tumorigenesis through the activation of the central signaling node $\mathrm{AKT}^{32,33}$, which is one of the most pivotal and versatile protein serine/threonine kinases at the core of glioma pathogenesis ${ }^{34,35}$. TRAF4 catalyzes the synthesis of the K63-linked polyubiquitin chain which recruits AKT to 

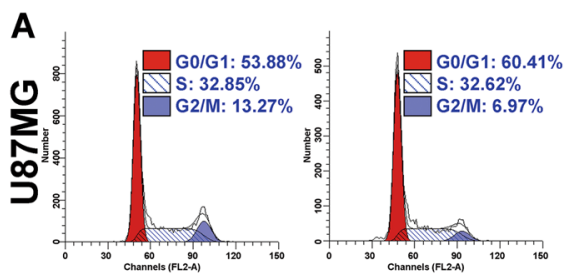

si-TRAF4\#1

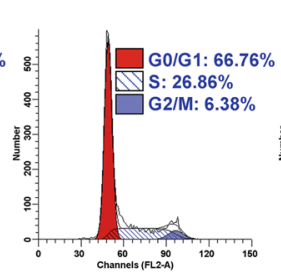

si-TRAF4\#2

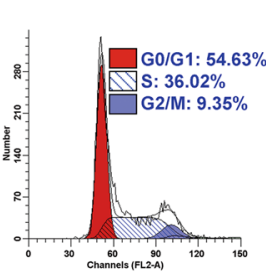

si-TRAF4+TRAF4

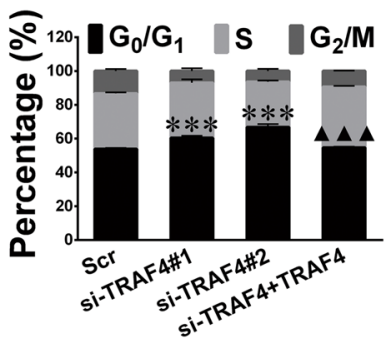

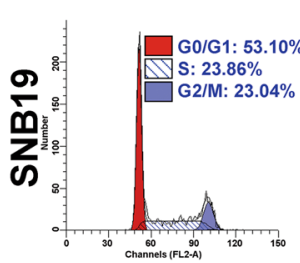

Scr

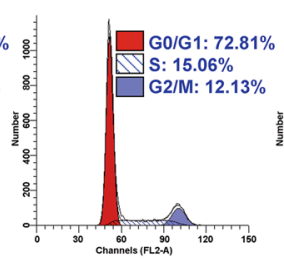

si-TRAF4\#1

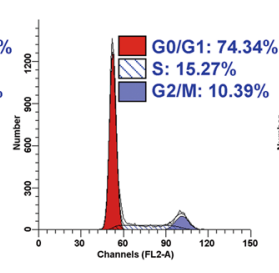

si-TRAF4\#2

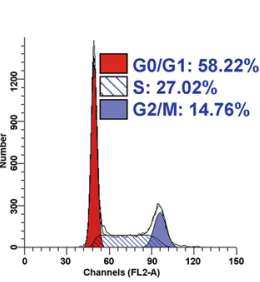

si-TRAF4+TRAF4

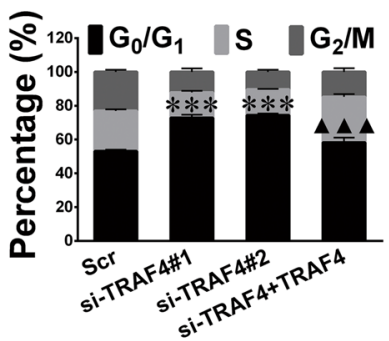

B

U87MG

SNB19
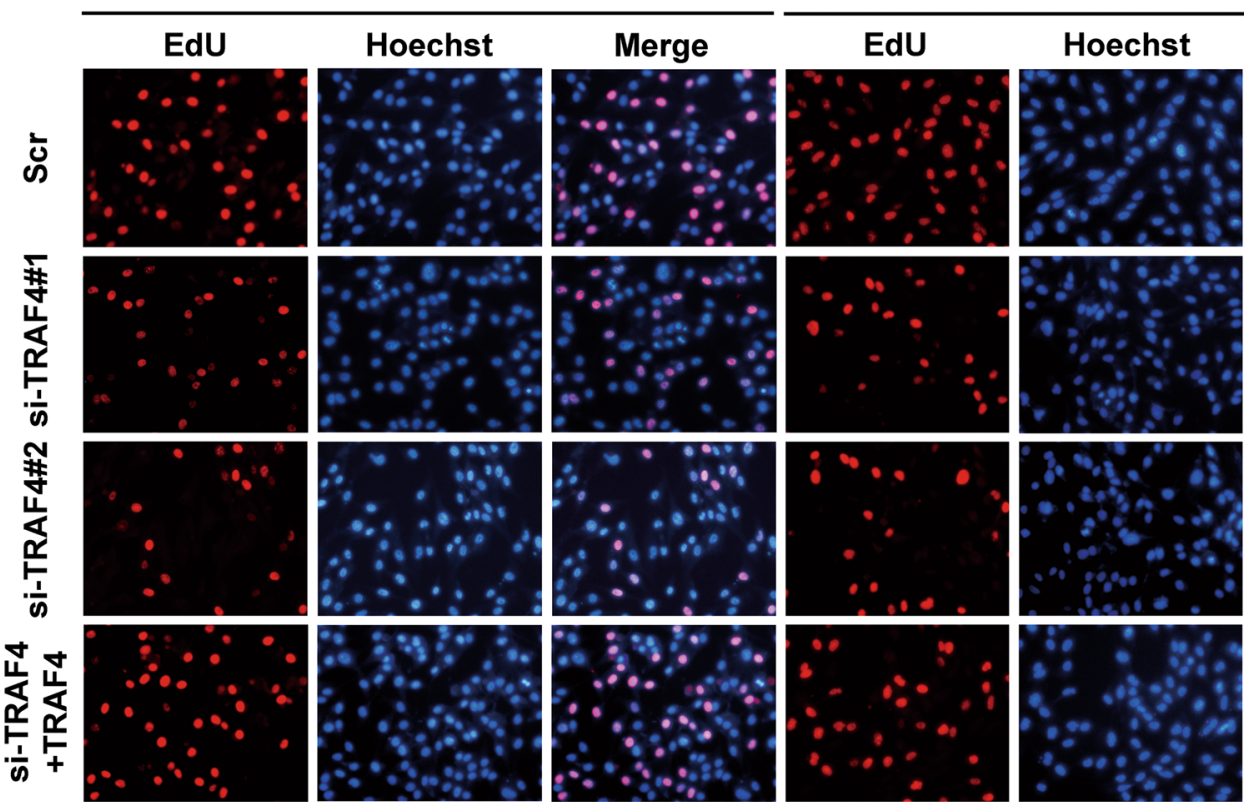

Merge
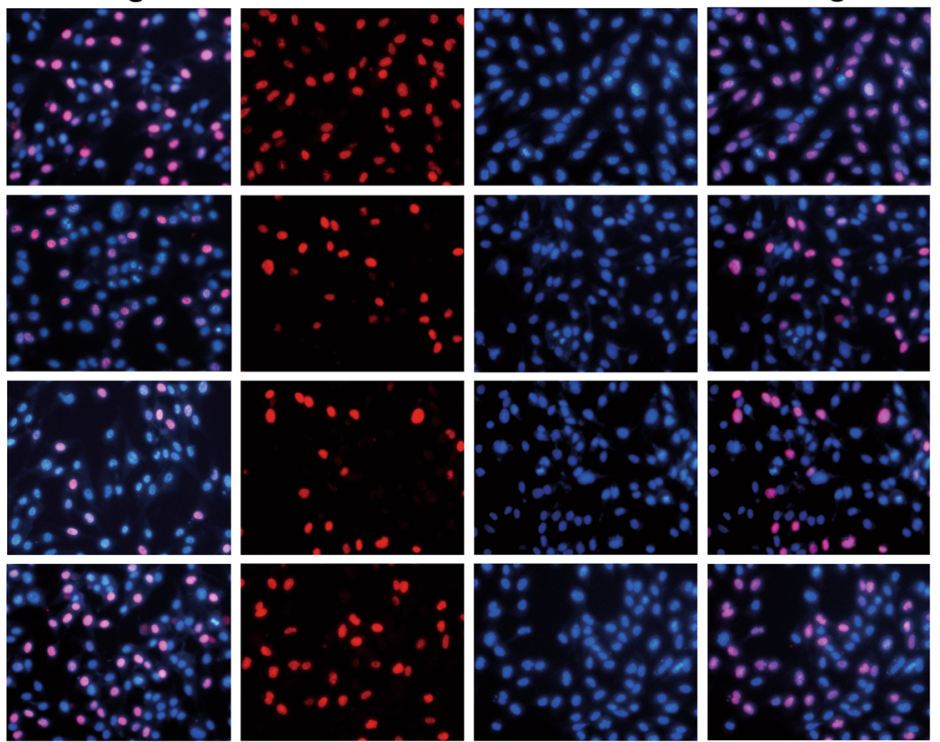

\section{C}

D

U87MG

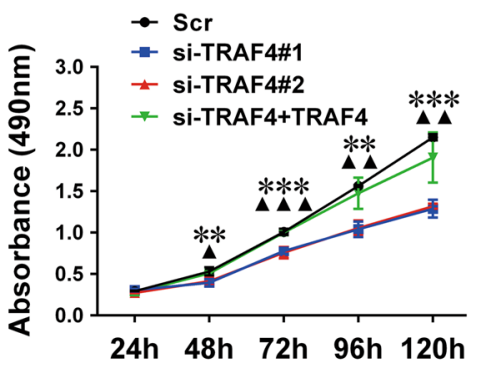

SNB19

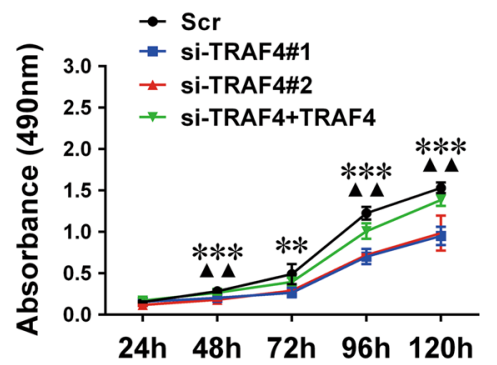

Fig. 7 Knockdown of endogenous TRAF4 suppresses cell cycle progression and glioma cell proliferation. a FCM cell cycle analysis results. U87MG and SNB19 cells were transfected with a scrambled control sequence (Scr) or TRAF4 siRNAs (si-TRAF4\#1 or si-TRAF4\#2) or the mixture of TRAF4 siRNAs plus the TRAF4 expression plasmid (si-TRAF4 + TRAF4). b, $\mathbf{c}$ EdU assay results (b) and quantitative analyses (c) of the cells as indicated. The EdU-positive and Hoechst-stained cells were counted as described in Fig. 2. $\mathbf{d}$ Growth curves of the indicated cells. All the experiments were

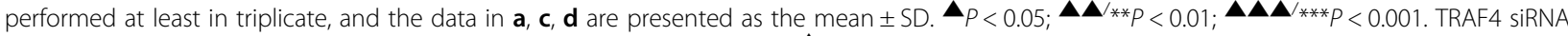
groups compared with the Scr group $\left(^{*}\right)$ or the si-TRAF4 + TRAF4 group $\left(\boldsymbol{\Delta}_{)}\right)$in a, d 


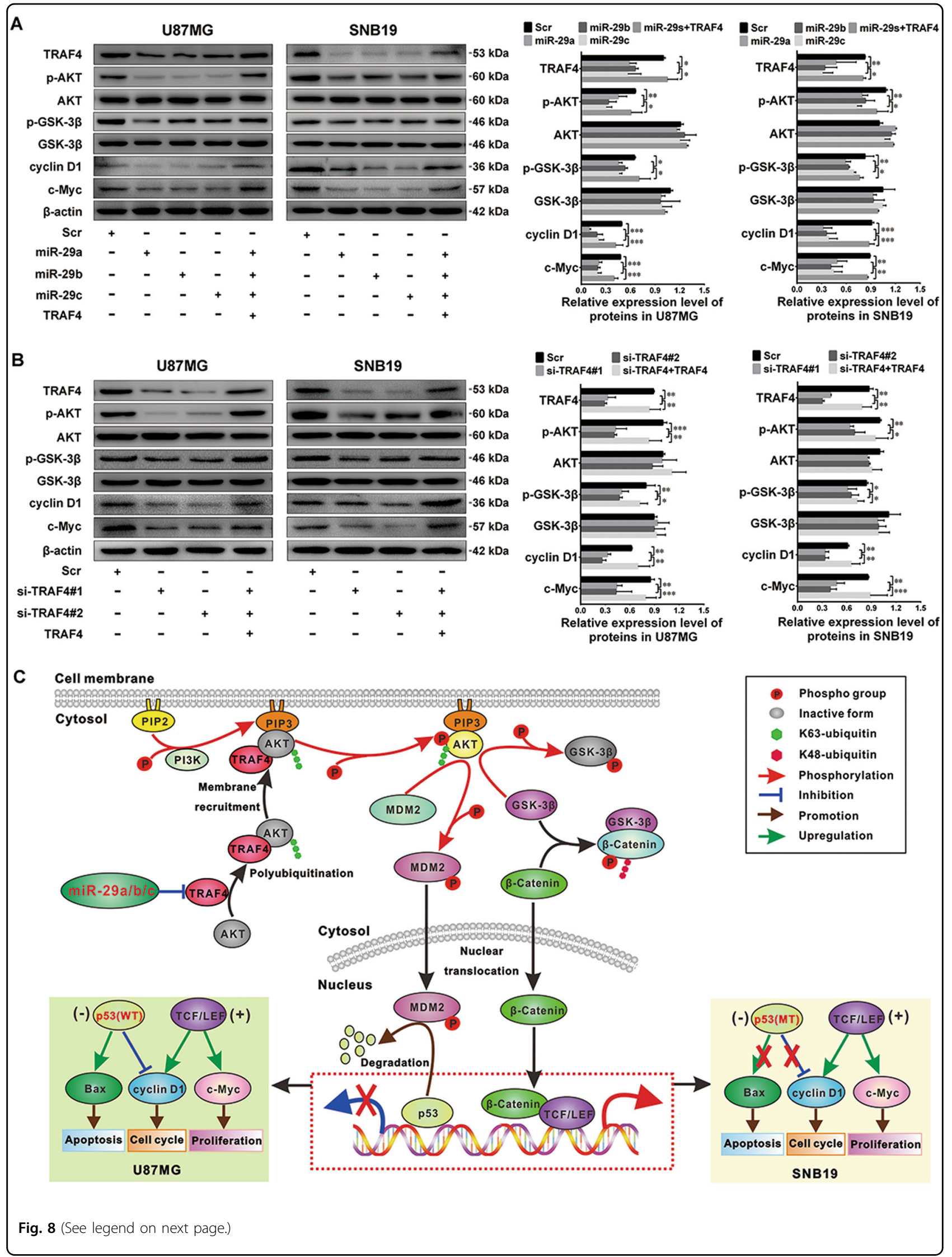


(see figure on previous page)

Fig. $8 \mathrm{miR}-29 \mathrm{a} / \mathrm{b} / \mathrm{c}$ and TRAF4 siRNA suppress the activation of the TRAF4/AKT/GSK-3 $\beta$ pathway. $\mathbf{a}, \mathbf{b}$ Western blot of TRAF4, p-AKT, AKT, p-

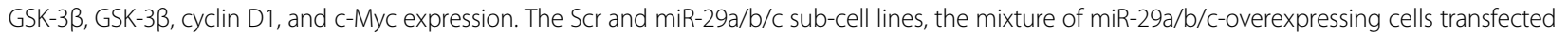
with the TRAF4 expression plasmid, the U87MG and SNB19 cells transfected with Scr or TRAF4 siRNAs (si-TRAF4\#1 or si-TRAF4\#2) or the mixture of TRAF4 siRNAs plus the TRAF4 expression plasmid were homogenized, and the total protein was extracted and subjected to western blot detection. All the experiments were performed at least in triplicate and the data in $\mathbf{a}, \mathbf{b}$ are presented as the mean $\pm \mathrm{SD}$. ${ }^{*} P<0.05$; ${ }^{* *} P<0.01$; ${ }^{* * *} P<0.001$. $\mathbf{c}$ Schematic illustration of the molecular pathways underlying the anti-glioma effects of miR-29a/b/c

the cell membrane, where AKT is phosphorylated and activated $^{32}$. Upon activation, AKT phosphorylates MDM2 and facilitates its translocation to cell nuclei. In cell nuclei, MDM2 induces the ubiquitination and degradation of both wild type and mutant p53 (refs. ${ }^{27,36-39}$ ) and thereby inhibits the transcription of $\mathrm{Bax}$ and cell apoptosis. In addition, GSK-3 $\beta$ is a critical component of the $\beta$-catenin destruction complex, as it captures, phosphorylates, and ubiquitinates cytoplasmic $\beta$-catenin. p-AKT catalyzes the inactivation of phosphorylated GSK-3 $\beta$, and thereby prevents $\beta$-catenin from proteasome mediated degradation $^{40-42}$. Free $\beta$-catenin then translocates to cell nuclei where it activates the transcription of $c-M y c$ and $c y c l i n D 1$ by forming a complex with the transcription factor TCF/ LET; this in turn accelerates G1/S-phase transition and cell proliferation ${ }^{40,43}$.

In the present study, although miR-29s-induced TRAF4 knockdown managed to block MDM2 phosphorylation and p53 degradation in both U87MG and SNB19 cell lines, it induced Bax expression and apoptosis only in U87MG with wild-type TP53. However, in SNB19 cells, since mutant p53 could not activate transcription of the $B a x$ gene, miR-29a/b/c failed to induce apoptosis in these cells. The DBTRG-05MG (TP53 wild-type) and U251 (TP53 mutant) cell lines were shown to be sensitive and resistant to miR-29s induced apoptosis, respectively, which was consistent with the findings discussed above. These results demonstrate that the pro-apoptotic effects of $\mathrm{miR}-29 \mathrm{a} / \mathrm{b} / \mathrm{c}$ require the presence of wild-type p53 and that $\mathrm{miR}-29 \mathrm{a} / \mathrm{b} / \mathrm{c}$ induce the apoptosis of glioma cells via the AKT/MDM2/p53 pathway in a p53-dependent manner (Fig. 8c). Moreover, we found that miR-29s-induced TRAF4 knockdown markedly blocked the phosphorylation of AKT and GSK-3 $\beta$ as well as the expression of cyclin D1 and c-Myc in glioma cells. Based on these findings, we conclude that miR-29a/b/c suppress G1/Sphase transition and the proliferation of glioma cells by targeting TRAF4 and down-modulating the AKT/GSK-3 $\beta$ pathway (Fig. 8c). These conclusions were also supported by siRNA simulation and TRAF4 rescue experiments.

In summary, our study indicates that $\mathrm{miR}-29 \mathrm{a} / \mathrm{b} / \mathrm{c}$ promote apoptosis and inhibit the proliferation of glioma cells by the direct targeting of TRAF4. The abnormal decrease in miR-29a/b/c is an important cause of TRAF4 overexpression and plays crucial roles in the tumorigenesis and malignant progression of gliomas. Our results underscore the practical values of these molecules as novel biomarkers for glioma grading and prognostic assessment, and imply that miR-29a/b/c and TRAF4 can be used as therapeutic candidates and as a target for malignant glioma, respectively.

\section{Bullet Points}

The expression of miR-29s decreases as the glioma grade increases and predicts worse patient prognosis.

Abnormal decreases in miR-29s are important factors that lead to TRAF4 overexpression

miR-29s induce glioma cell apoptosis in a p53dependent manner through the TRAF4/AKT/MDM2 pathway.

miR-29s restrain cell proliferation by directly targeting the TRAF4/AKT/GSK-3 $\beta$ pathway.

miR-29s and TRAF4 are potential biomarkers for glioma grading and prognosis.

\section{Acknowledgements}

This research was supported by the National Natural Science Foundation of China (No. 81872061, 81402050, 81502166, and 81672592), the Program of Science and Technology of Tianjin Municipality (No. 15JCYBJC49900, 15JCZDJC34600, 16JCQNJC13400, and 17JCYBJC27100), the Program of Tianjin Municipal Health Bureau (No. 15KJ147), the Foundation of Tianjin Medical University and General Hospital (No. 2015KYZQ11 and ZYYFY2015032), and the "Junior Technical Backbone" Training Project of TMUGH (2016, to C. Shi).

\section{Author details \\ ${ }^{1}$ Department of Neuropathology, Tianjin Neurological Institute, Tianjin Medical University General Hospital, Tianjin 300052, China. ${ }^{2}$ Tianjin Key Laboratory of Injuries, Variations and Regeneration of the Nervous System, Tianjin 300052, China. ${ }^{3}$ Key Laboratory of Post-trauma Neuro-Repair and Regeneration in Central Nervous System, Ministry of Education, Tianjin 300052, China. ${ }^{4}$ Department of Biochemistry and Molecular Biology, School of Basic Medical Sciences of Tianjin Medical University, Tianjin 300070, China. ${ }^{5}$ Lab of Neuro- Oncology, Tianjin Neurological Institute, Tianjin Medical University General Hospital, Tianjin 300052, China}

\section{Conflict of interest}

The authors declare that they have no conflict of interest.

\section{Publisher's note}

Springer Nature remains neutral with regard to jurisdictional claims in published maps and institutional affiliations.

Supplementary Information accompanies this paper at (https://doi.org/ 10.1038/s41419-018-1092-x). 
Received: 21 May 2018 Revised: 19 September 2018 Accepted: 21 September 2018

Published online: 22 October 2018

\section{References}

1. Louis, D. N. et al. The 2016 World Health Organization Classification of Tumors of the Central Nervous System: a summary. Acta Neuropathol. 131, 803-820 (2016).

2. Ricard, D. et al. Primary brain tumours in adults. Lancet 379, 1984-1996 (2012)

3. Wen, P. Y. \& Kesari, S. Malignant gliomas in adults. N. Engl. J. Med 359, 492-507 (2008).

4. Stupp, R. et al. Radiotherapy plus concomitant and adjuvant temozolomide for glioblastoma. N. Engl. J. Med. 352, 987-996 (2005).

5. Riddick, G. \& Fine, H. A. Integration and analysis of genome-scale data from gliomas. Nat. Rev. Neurol. 7, 439-450 (2011).

6. Li, Y. et al. miR-146b-5p inhibits glioma migration and invasion by targeting MMP16. Cancer Lett. 339, 260-269 (2013).

7. Liu, J. et al. miR-146b-5p functions as a tumor suppressor by targeting TRAF6 and predicts the prognosis of human gliomas. Oncotarget 6, 29129-29142 (2015).

8. Shi, Y. et al. miR-663 suppresses oncogenic function of CXCR4 in glioblastoma Clin. Cancer Res. 21, 4004-4013 (2015).

9. $\mathrm{Li}, \mathrm{H}$. et al. miR-320a functions as a suppressor for gliomas by targeting SND1 and beta-catenin, and predicts the prognosis of patients. Oncotarget 8 , 19723-19737 (2017)

10. Shi, C. et al. miR-29a/b/c function as invasion suppressors for gliomas by targeting CDC42 and predict the prognosis of patients. Br. J. Cancer 117 1036-1047 (2017).

11. Lagos-Quintana, M., Rauhut, R., Lendeckel, W. \& Tuschl, T. Identification of novel genes coding for small expressed RNAs. Science 294, 853-858 (2001).

12. Cushing, L. et al. Disruption of miR-29 leads to aberrant differentiation of smooth muscle cells selectively associated with distal lung vasculature. PLoS Genet. 11, e1005238 (2015).

13. Kwon, J. J. et al. Pathophysiological role of microRNA-29 in pancreatic cancer stroma. Sci. Rep. 5, 11450 (2015).

14. Fabbri, M. et al. MicroRNA-29 family reverts aberrant methylation in lung cancer by targeting DNA methyltransferases 3A and 3B. Proc. Natl Acad. Sci. USA 104, 15805-15810 (2007).

15. Xiong, $Y$. et al. Effects of microRNA-29 on apoptosis, tumorigenicity, and prognosis of hepatocellular carcinoma. Hepatology 51, 836-845 (2010).

16. Cummins, J. M. et al. The colorectal microRNAome. Proc. Natl Acad. Sci. USA 103, 3687-3692 (2006).

17. Garzon, R. et al. MicroRNA 29b functions in acute myeloid leukemia. Blood 114, 5331-5341 (2009).

18. Xi, Z et al. Overexpression of miR-29a reduces the oncogenic properties of glioblastoma stem cells by downregulating Quaking gene isoform 6. Oncotarget 8, 24949-24963 (2017).

19. Sun, G. et al. MiR-29b inhibits the growth of glioma via MYCN dependent way. Oncotarget 8, 45224-45233 (2017).

20. Zhao, J. J. et al. microRNA expression profile and identification of miR-29 as a prognostic marker and pathogenetic factor by targeting CDK6 in mantle cell lymphoma. Blood 115, 2630-2639 (2010).
21. Huang, $X$. et al. Targeted delivery of microRNA-29b by transferrin-conjugated anionic lipopolyplex nanoparticles: a novel therapeutic strategy in acute myeloid leukemia. Clin. Cancer Res. 19, 2355-2367 (2013).

22. Camilleri-Broet, S. et al. TRAF4 overexpression is a common characteristic of human carcinomas. Oncogene 26, 142-147 (2007).

23. Rhodes, D. R. et al. Large-scale meta-analysis of cancer microarray data identifies common transcriptional profiles of neoplastic transformation and progression. Proc. Natl Acad. Sci. USA 101, 9309-9314 (2004).

24. Ahmed, F., Shiraishi, T., Vessella, R. L. \& Kulkarni, P. Tumor necrosis factor receptor associated factor-4: an adapter protein overexpressed in metastatic prostate cancer is regulated by microRNA-29a. Oncol. Rep. 30, 2963-2968 (2013).

25. Cushing, L. et al. miR-29 is a major regulator of genes associated with pulmonary fibrosis. Am. J. Respir. Cell Mol. Biol. 45, 287-294 (2011).

26. Forbes, S. et al. Cosmic 2005. Br. J. Cancer 94, 318-322 (2006).

27. Li, $Y$., et al. PTEN has tumor-promoting properties in the setting of gain-offunction p53 mutations. Cancer Res. 68, 1723-1731 (2008).

28. Kruse, C. A. et al. Characterization of a continuous human glioma cell line DBTRG-05MG: growth kinetics, karyotype, receptor expression, and tumor suppressor gene analyses. In Vitro Cell Dev. Biol. 28A, 609-614 (1992).

29. Fujiwara, T. et al. Retroviral-mediated transduction of p53 gene increases TGFbeta expression in a human glioblastoma cell line. Int J. Cancer 56, 834-839 (1994).

30. Leroy, B. et al. Analysis of TP53 mutation status in human cancer cell lines: a reassessment. Hum. Mutat. 35, 756-765 (2014).

31. Furnari, F. B. et al. Malignant astrocytic glioma: genetics, biology, and paths to treatment. Genes Dev. 21, 2683-2710 (2007).

32. Li, W. et al. TRAF4 is a critical molecule for Akt activation in lung cancer. Cancer Res. 73, 6938-6950 (2013).

33. Zhang, J., Li, X., Yang, W., Jiang, X. \& Li, N. TRAF4 promotes tumorigenesis of breast cancer through activation of Akt. Oncol. Rep. 32, 1312-1318 (2014)

34. Holland, E. C. et al. Combined activation of Ras and Akt in neural progenitors induces glioblastoma formation in mice. Nat. Genet. 25, 55-57 (2000).

35. Chautard, E., Ouedraogo, Z. G., Biau, J. \& Verrelle, P. Role of Akt in human malignant glioma: from oncogenesis to tumor aggressiveness. J. Neurooncol. 117, 205-215 (2014)

36. Zhou, B. P. et al. HER-2/neu induces p53 ubiquitination via Akt-mediated MDM2 phosphorylation. Nat. Cell Biol. 3, 973-982 (2001).

37. Mayo, L. D. \& Donner, D. B. A phosphatidylinositol 3-kinase/Akt pathway promotes translocation of Mdm2 from the cytoplasm to the nucleus. Proc. Natl Acad. Sci. USA 98, 11598-11603 (2001).

38. Abraham, A. G. \& O'Neill, E. PI3K/Akt-mediated regulation of p53 in cancer. Biochem. Soc. Trans. 42, 798-803 (2014).

39. Terzian, T. et al. The inherent instability of mutant p53 is alleviated by Mdm2 or p16INK4a loss. Genes Dev. 22, 1337-1344 (2008).

40. McCubrey, J. A. et al. Multifaceted roles of GSK-3 and Wnt/beta-catenin in hematopoiesis and leukemogenesis: opportunities for therapeutic intervention. Leukemia 28, 15-33 (2014).

41. Tauriello, D. V. \& Maurice, M. M. The various roles of ubiquitin in Wnt pathway regulation. Cell Cycle 9, 3700-3709 (2010).

42. Freyberg, Z., Ferrando, S. J. \& Javitch, J. A. Roles of the Akt/GSK-3 and Wnt signaling pathways in schizophrenia and antipsychotic drug action. Am. J. Psychiatry 167, 388-396 (2010).

43. Manning, B. D. \& Cantley, L. C. AKT/PKB signaling: navigating downstream. Cell 129, 1261-1274 (2007). 